\title{
2-LINEAR OPERATORS ON 2-MODULAR SPACES
}

\author{
Burhanudin Arif Nurnugroho $^{1}$, Supama ${ }^{2, *}$ and Atok Zulijanto ${ }^{3}$ \\ ${ }^{1}$ Department of Mathematical Education \\ Ahmad Dahlan University \\ Yogyakarta, Indonesia \\ e-mail: burhanarifmath@gmail.com \\ 1,2,3 Department of Mathematics \\ Universitas Gadjah Mada \\ Yogyakarta 55281, Indonesia \\ e-mail: supama@ugm.ac.id \\ atokzulijanto@ugm.ac.id
}

\begin{abstract}
In this paper, we observe some topological properties of 2-modular spaces. Further, we introduce and characterize a 2- $\rho$-bounded 2-linear operator from a 2-modular space into a normed space as well.
\end{abstract}

\section{Introduction and Preliminaries}

A modular space has important roles and applications in many areas, such as engineering, physics, economics, social sciences, etc. Therefore, it gains a lot of attention of many researchers from many fields. A concept of

Received: July 7, 2017; Revised: July 22, 2017; Accepted: October 2, 2017

2010 Mathematics Subject Classification: 46A80, 47Axx.

Keywords and phrases: 2-norm, 2-modular, 2-linear operator.

${ }^{*}$ Corresponding author

Communicated by Choonkil Park; Editor: International Journal of Functional Analysis, Operator Theory and Applications: Published by Pushpa Publishing House, Allahabad, India. 
3194 Burhanudin Arif Nurnugroho, Supama and Atok Zulijanto

modular spaces was firstly initiated by Nakano in 1950 (see [6, 8, 12]). Later on, Mazur and Orlicz [7] and Musielak and Orlicz [9] modified the definition of the modular space proposed by Nakano, by avoiding the lattice structure in the space $X$ on which the modular is defined as well as the monotonicity axiom for the modular.

As usual, the symbols $\mathbb{N}, \mathbb{R}$ and $\mathbb{R}^{*}$ denote the natural number system, the real number system and the extended real number system, respectively. As given in [9], we can rewrite the definition of the modular as the following. Let $X$ be a real linear space over $\mathbb{R}$. A nonnegative function $\rho: X \rightarrow \mathbb{R}^{*}$ is called a modular if for every $x, y \in X$, the following conditions hold:

(i) $\rho(x)=0$ if and only if $x=0$,

(ii) $\rho(-x)=\rho(x)$, and

(iii) $\rho(\alpha x+\beta y) \leq \rho(x)+\rho(y)$ for every $\alpha, \beta \geq 0$ with $\alpha+\beta=1$.

If the condition (iii) is replaced by

(iii') $\rho(\alpha x+\beta y) \leq \alpha \rho(x)+\beta \rho(y)$ for every $\alpha, \beta \geq 0$ with $\alpha+\beta=1$, then the modular $\rho$ is called a convex modular. A real linear space $X$ equipped with a modular $\rho$, written $(X, \rho)$ or $X$ in short, is called a modular space.

Based on the definition of a modular as given above, we can easily check that every norm is a modular. Therefore, we can consider a modular as a generalization of a norm. As consequences, many concepts in normed spaces can be generalized into modular spaces.

In an earlier paper ([2] and [3]), Gahler introduced a concept of 2-norm spaces and $n$-norm spaces. One knows that every $n$-norm can define an $n-1$-norm. See [4] and [5]. Inductively, from an $n$-norm, we can derive a norm. Further, based on the theory of Gahler, Chu et al. [1] characterized 2-isometries on 2-norm spaces. Srivastava et al. [11] characterized linear 
$n$-functionals in $n$-norm spaces. Moreover, they formulated the extension of Hanh-Banach theorem for linear $n$-functionals in $n$-norm spaces.

Modular spaces are closed related to normed spaces [12]. Meanwhile, as mentioned before, any $n$-norm can define a norm $([4,5])$. Based on these facts and analogously to the definition of an $n$-norm, Nourouzi and Shabanian [10] defined a notion of $n$-modular spaces. In the present paper, we observe some topological properties of 2-modular spaces. We also introduce a definition of a 2- $\rho$-bounded 2-linear operator from a 2-modular space into a normed space. Furthermore, some properties of a 2- $\rho$-bounded 2-linear operator from a 2-modular space into a normed space are observed as well.

\section{2-modular Spaces}

As usual, symbols $\mathbb{N}, \mathbb{R}$ and $\mathbb{R}^{*}$ denote a natural numbers system, a real number system and an extended real numbers system, respectively. For any linear space $X, \operatorname{dim}(X)$ means the dimension of $X$. In this paper, we always assume that for any linear space $X$, the $\operatorname{dim}(X) \geq 2$, unless otherwise mentioned.

Further, we give a definition of a 2-modular, analogously with those of a 2-norm.

Definition 2.1. Let $X$ be a real linear space with $\operatorname{dim}(X) \geq 2$. A real valued function $\rho(\cdot, \cdot): X \times X \rightarrow \mathbb{R}^{*}$ is called a 2-modular on $X$ if

(i) $\rho(x, y)=0$ if and only if $x$ and $y$ are linearly dependent,

(ii) $\rho(x, y)=\rho(y, x)$ for every $x, y \in X$,

(iii) $\rho(-x, y)=\rho(y, x)$ for every $x, y \in X$, and

(iv) $\rho(\alpha x+\beta y, z) \leq \rho(x, z)+\rho(y, z)$ for every $x, y, z \in X$ and for every $\alpha, \beta \geq 0$ with $\alpha+\beta=1$. 
3196 Burhanudin Arif Nurnugroho, Supama and Atok Zulijanto

If the condition (iv) is replaced by

(iv') $\rho(\alpha x+\beta y, z) \leq \alpha \rho(x, z)+\beta \rho(y, z)$ for every $x, y, z \in X$ and for every $\alpha, \beta \geq 0$ with $\alpha+\beta=1$,

then $\rho(\cdot, \cdot)$ is called a convex 2-modular.

It is easy to prove that $\rho(x, y) \geq 0$ for every $x, y \in X$. Moreover, following the condition (i) in Definition 2.1, we have

(i) $\rho(x, 0)=0$ for every $x \in X$, and

(ii) if $\rho(x, y)=0$ for every $y \in X$, then $x=0$.

Following are examples of 2-modulars.

Example 2.2. Let $X=\mathbb{R}^{2}$. If the function $\rho: X \times X \rightarrow \mathbb{R}^{*}$ is defined by

$$
\rho(x, y)=\operatorname{abs}\left(\left|\begin{array}{ll}
x_{1} & x_{2} \\
y_{1} & y_{2}
\end{array}\right|\right),
$$

then $\rho$ is a 2-modular on $X$.

Example 2.3. Let $X$ be a real linear space and $\|\cdot, \cdot\|$ a 2-norm on $X$. Then

$$
\rho(x, y)=\int_{0}^{\|x, y\|}\left(e^{t}-1\right) d t
$$

is a 2-modular on $X$.

It can be seen that every 2-norm on a linear space $X$ is a 2-modular, but the converse is not true.

Example 2.4. Let $X=\mathbb{R}^{2}$. If the function $\rho: X \times X \rightarrow \mathbb{R}^{*}$ is defined by

$$
\rho(x, y)=\sqrt{\operatorname{abs}\left(\left|\begin{array}{ll}
x_{1} & x_{2} \\
y_{1} & y_{2}
\end{array}\right|\right)},
$$

then $\rho$ is a 2-modular on $X$. However, $\rho$ is not a 2-norm on $X$. 
Theorem 2.5. Any 2-modular on a real linear space $X$ generates $a$ modular on $X$.

Proof. Let $\rho(\cdot, \cdot)$ be a 2-modular on a real linear space $X$. Take any linearly independent set of vectors $\left\{a_{1}, a_{2}\right\}$ on $X$. Define a function $\sigma: X \rightarrow \mathbb{R}^{*}$ by

$$
\sigma(x)=\max \left\{\rho\left(x, a_{1}\right), \rho\left(x, a_{2}\right)\right\},
$$

then $\sigma(-x)=\sigma(x)$ for every $x \in X$ and

$$
\begin{aligned}
\sigma(x) & =0 \Leftrightarrow \max \left\{\rho\left(x, a_{1}\right), \rho\left(x, a_{2}\right)\right\} \\
& \Leftrightarrow \rho\left(x, a_{1}\right)=\rho\left(x, a_{2}\right)=0 \\
& \Leftrightarrow\left\{x, a_{1}\right\} \text { and }\left\{x, a_{2}\right\} \text { are linearly dependent } \\
& \Leftrightarrow x=0,
\end{aligned}
$$

since $\left\{a_{1}, a_{2}\right\}$ is linearly independent. Now, let $x, y \in X$ and $\alpha, \beta \geq 0$ be such that $\alpha+\beta=1$. Then

$$
\begin{aligned}
\sigma(\alpha x+\beta y) & =\max \left\{\rho\left(\alpha x+\beta y, a_{1}\right), \rho\left(\alpha x+\beta y, a_{2}\right)\right\} \\
& \leq \max \left\{\rho\left(x, a_{1}\right), \rho\left(x, a_{2}\right)\right\}+\max \left\{\rho\left(y, a_{1}\right), \rho\left(y, a_{2}\right)\right\} \\
& =\sigma(x)+\sigma(y) .
\end{aligned}
$$

Thus, the function $\sigma$ is a modular.

The following theorem describes some basic properties of a 2-modular.

Theorem 2.6. If $\rho$ is a 2-modular on a real linear space $X$, then

(i) $\rho(\lambda x, y) \leq \rho(x, y)$ for every $x, y \in X$ and $|\lambda| \leq 1$.

(ii) $\rho\left(\sum_{k=1}^{n} \lambda_{k} x_{k}, y\right) \leq \sum_{k=1}^{n} \rho\left(x_{k}, y\right)$ for every $x_{k}, \quad y \in X$ and $\lambda_{k} \geq 0, k=1,2, \ldots, n$, with $\sum_{k=1}^{n} \lambda_{k}=1$. 
(iii) $\rho(\alpha x, y) \leq \rho(\beta x, y)$ for every $x, y \in X$ and $\alpha, \beta \in \mathbb{R}$ with $0<\alpha \leq \beta$.

Proof. (i) It is trivial for $\lambda=0$ or $|\lambda|=1$. Now, let $0<\lambda<1$. Then

$$
\rho(\lambda x, y)=\rho(\lambda x+(1-\lambda) 0, y) \leq \rho(x, y) .
$$

Moreover, following the condition (iii) in Definition 2.1, then we have

$$
\rho(\lambda x, y) \leq \rho(x, y)
$$

for every $-1<\lambda<0$. So, (i) is proved.

(ii) We are going to prove (ii) by mathematical induction. It is true for $x_{1}, x_{2}, y$ and $\lambda_{1}, \lambda_{2} \geq 0$ with $\lambda_{1}+\lambda_{2}=1$, because of the condition (iv) in Definition 2.1. Assume that it is true for $x_{1}, x_{2}, \ldots, x_{n}, y$ and $\lambda_{1}, \lambda_{2}, \ldots, \lambda_{n}$ $\geq 0$ with $\sum_{k=1}^{n} \lambda_{k}=1$. Then

$$
\rho\left(\sum_{k=1}^{n} \lambda_{k} x_{k}, y\right) \leq \sum_{k=1}^{n} \rho\left(x_{k}, y\right) .
$$

Now, take any $x_{1}, x_{2}, \ldots, x_{n+1}, y \in X$ and $\lambda_{1}, \lambda_{2}, \ldots, \lambda_{n+1} \geq 0$ such that $\sum_{k=1}^{n+1} \lambda_{k}=1$, then there is a positive integer $j, 1 \leq j \leq n+1$, such that $\lambda_{j} \neq 0$. So, we have

$$
\begin{aligned}
\rho\left(\sum_{k=1}^{n+1} \lambda_{k} x_{k}, y\right) & =\rho\left(\left(1-\lambda_{j}\right) \sum_{k=1, k \neq j}^{n+1} \frac{\lambda_{k} x_{k}}{1-\lambda_{j}}+\lambda_{j} x_{j}, y\right) \\
& \leq \rho\left(\left(1-\lambda_{j}\right) \sum_{k=1, k \neq j}^{n+1} \frac{\lambda_{k} x_{k}}{1-\lambda_{j}}, y\right)+\rho\left(x_{j}, y\right) \\
& \leq \sum_{k=1, k \neq j}^{n+1} \rho\left(x_{k}, y\right)+\rho\left(x_{j}, y\right)=\sum_{k=1}^{n+1} \rho\left(x_{k}, y\right) .
\end{aligned}
$$


(iii) Following condition (iv) in Definition 2.1, then the assertion follows.

Let $X$ be a real linear space. A 2-modular $\rho$ on $X$ is said to satisfy the $\Delta_{2}$-condition if there exists a constant $K>0$ such that $\rho(2 x, y) \leq K \rho(x, y)$ for every $x, y \in X$. The 2-modular $\rho$ as given in Example 2.4 satisfies the $\Delta_{2}$-condition. However, the 2-modular $\rho$ as given in Example 2.3 does not satisfy the $\Delta_{2}$-condition.

Throughout this paper, we always assume that the 2-modular $\rho$ satisfies the $\Delta_{2}$-condition.

Let $\rho$ be a 2-modular on a real linear space $X$. We define

$$
X_{\rho}=\{x \in X: \rho(\lambda x, y)<\infty \text {, for some } \lambda>0 \text { and for any } y \in X\} \text {. }
$$

It can easily be proved that $X_{\rho}$ is a real linear space. Moreover, $X_{\rho}$ is a 2 -modular space with respect to $\rho$. We can also prove that $\rho(x, y)<\infty$ for every $x \in X_{\rho}$ and for every $y \in X$.

Throughout this paper, $X_{\rho}$ is always meant as given in (2.1).

\section{Topological Properties of 2-modular Spaces}

In this section, we introduce some topological concept with respect to a 2-modular. We begin our discussion by giving a notion of 2-modular convergent sequences in the space $X_{\rho}$.

Let $X_{\rho}$ be a 2-modular space. A sequence $\left\{x_{n}\right\}$ in $X_{\rho}$ is said to be 2-modular convergent (or $\rho$-convergent) to some $x \in X_{\rho}$, denoted by

$$
\rho-\lim x_{n}=x,
$$

if for every $y \in X_{\rho}, \lim \rho\left(x_{n}-x, y\right)=0$, i.e., for every $\varepsilon>0$, there exists an $N \in \mathbb{N}$ such that for any integer $n \geq N$, we have $\rho\left(x_{n}-x, y\right)<\varepsilon$. In 
this case, the vector $x$ is called a 2-modular limit ( $\rho$-limit) of the sequence $\left\{x_{n}\right\}$.

Example 3.1. Let $X$ and $\rho$ be as given in Example 2.4. It is clear that $X_{\rho}=X$. Let $x_{n}=\left(\frac{1}{n}, 0\right)$ for every $n \in \mathbb{N}$ and $x=(0,0)$. For any $y=\left(y_{1}, y_{2}\right) \in X$, we have

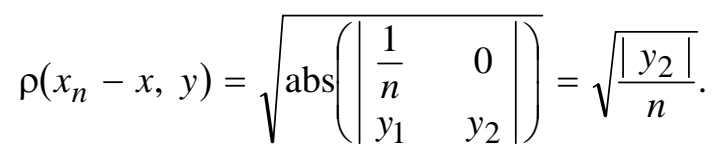

Given $\varepsilon>0$, we can choose a positive integer $N$ such that $\sqrt{\frac{\left|y_{2}\right|}{N}}<\varepsilon$. Hence, the sequence $\left\{x_{n}\right\} \rho$-converges to $x$.

We observe some basic properties of the $\rho$-convergence of a sequence in any 2-modular space. Let us see the following theorems:

Theorem 3.2. Let $X_{\rho}$ be a 2-modular space and $\left\{x_{n}\right\}$ be a sequence in $X_{\rho}$. If $\left\{x_{n}\right\}$ is $\rho$-convergent, then its $\rho$-limit is unique.

Proof. Since the 2-modular $\rho$ satisfies the $\Delta_{2}$-condition, there exists a constant $K>0$ such that

$$
\rho(2 x, y) \leq K \rho(x, y),
$$

for every $x, y \in X_{\rho}$. Given any $\varepsilon>0$. Suppose $\left\{x_{n}\right\} \rho$-converges to $x$ and $z$ in $X_{\rho}$. For any $y \in X_{\rho}$, there exists an $N \in \mathbb{N}$ such that

$$
\rho\left(x_{N}-x, y\right)<\frac{\varepsilon}{2 K} \quad \text { and } \rho\left(x_{N}-z, y\right)<\frac{\varepsilon}{2 K} .
$$

These imply

$$
\rho(x-z, y) \leq \rho\left(2\left(x_{N}-x, y\right)\right)+\rho\left(2\left(x_{N}-z, y\right)\right)<\varepsilon .
$$

Since the expression (3.1) holds for any $\varepsilon>0$, we obtain $\rho(x-z, y)=0$ for every $y \in X_{\rho}$. This implies $x=z$. 
Theorem 3.3. Let $X_{\rho}$ be a 2-modular space and $\left\{x_{n}\right\}$ be a sequence in $X_{\rho}$. If for every $z \in X_{\rho}, \lim \rho\left(x_{n}-x, z\right)=\lim \rho\left(x_{n}-y, z\right)=0$ for some $x, y \in X_{\rho}$, then

(i) $\rho\left(\alpha x_{n}-\alpha x, z\right)=0$ for every real number $\alpha$, and

(ii) $\rho\left(\left(x_{n}+y_{n}\right)-(x+y, z)\right)=0$.

Proof. Since the 2-modular $\rho$ satisfies the $\Delta_{2}$-condition, there exists a constant $K>0$ such that $\rho(2 x, y) \leq K \rho(x, y)$ for every $x, y \in X_{\rho}$.

(i) It is trivial for $\alpha=0$. Let $\alpha>0$ be an arbitrary, there is a positive integer $p$ such that $\alpha<2^{p}$. Given $\varepsilon>0$. Since $\rho\left(x_{n}-x, z\right)=0$, there exists an $N \in \mathbb{N}$ such that for every $n \geq N$, we have $\rho\left(x_{n}-x, z\right)<\frac{\varepsilon}{K^{p}}$. This implies

$$
\rho\left(\alpha x_{n}-\alpha x, z\right) \leq \rho\left(2^{p}\left(x_{n}-x\right), z\right) \leq K^{p} \rho\left(x_{n}-x, z\right)<\varepsilon .
$$

In other words, $\lim \rho\left(\alpha x_{n}-\alpha x, z\right)=0$. Moreover, following the condition (iii) in Definition 2.1, we obtain $\lim \rho\left(\alpha x_{n}-\alpha x, z\right)=0$ for every $\alpha \in \mathbb{R}$.

(ii) Since

$$
\begin{aligned}
\rho\left(\left(x_{n}+y_{n}\right)-(x+y), z\right) & \leq \rho\left(2\left(x_{n}-x\right), z\right)+\rho\left(2\left(y_{n}-y\right), z\right) \\
& \leq K\left(\rho\left(x_{n}-x, z\right)+\rho\left(y_{n}-y, z\right)\right),
\end{aligned}
$$

the assertion follows.

A sequence $\left\{x_{n}\right\}$ in $X_{\rho}$ is called a $\rho$-Cauchy sequence if for every $\varepsilon>0$, there is a positive integer $N$ such that

$$
\rho\left(x_{n}-x_{m}, y\right)<\varepsilon,
$$

for every $m, n \geq N$. The correlation between $\rho$-convergent and $\rho$-Cauchy sequences is formulated in the following theorem: 
Theorem 3.4. Every $\rho$-convergent sequence in $X_{\rho}$ is a $\rho$-Cauchy sequence.

Proof. We can choose a constant $K>0$ such that $\rho(2 x, y) \leq K \rho(x, y)$ for all $x, y \in X_{\rho}$, since the 2-modular $\rho$ satisfies the $\Delta_{2}$-condition. Now, let $\left\{x_{n}\right\}$ be any sequence in $X_{\rho}$ that $\rho$-converges, say to some $x \in X_{\rho}$. Given any $\varepsilon>0$ and $y \in X_{r h o}$, then there is a positive integer $N$ such that $\rho\left(x_{n}-x, y\right)<\frac{\varepsilon}{3 K}$ for every $n \geq N$. Further, for any $m, n \geq N$, we have

$$
\begin{aligned}
\rho\left(x_{n}-x_{m}, y\right) & \leq \rho\left(2\left(x_{n}-x\right), y\right)+\rho\left(2\left(x-x_{m}\right), y\right) \\
& \leq K\left(\rho\left(x_{n}-x, y\right)+\rho\left(x_{m}-x, y\right)\right)<\varepsilon .
\end{aligned}
$$

So, the proof is complete.

We also characterize $\rho$-Cauchy sequences, as given in the following theorem:

Theorem 3.5. A sequence $\left\{x_{n}\right\}$ in $X_{\rho}$ is $\rho$-Cauchy if and only if $\left\{\alpha x_{n}\right\}$ is a $\rho$-Cauchy sequence for all $\alpha \in \mathbb{R}$.

Proof. $(\Leftarrow$ :) By taking $\alpha=1$, the assertion follows.

$(\Rightarrow$ :) It is trivial for $\alpha=0$. Let $\alpha>0$ be an arbitrary. Then there is a positive integer $p$ such that $\alpha<2^{p}$. Since the 2-modular $\rho$ satisfies the $\Delta_{2}$ condition, there is a constant $K>0$ such that $\rho(2 x, y) \leq K \rho(x, y)$ for all $x, y \in X_{\rho}$.

Let $\left\{x_{n}\right\}$ be a $\rho$-Cauchy sequence. Given $\varepsilon>0$ and $y \in X_{\rho}$, there exists an $N \in \mathbb{N}$ such that for every $m, n \geq N$, we have $\rho\left(x_{n}-x_{m}, y\right)<\frac{\varepsilon}{K^{p}}$. This implies

$$
\rho\left(\alpha x_{n}-\alpha x_{m}, y\right) \leq \rho\left(2^{p}\left(x_{n}-x_{m}\right), y\right) \leq K^{p} \rho\left(x_{n}-x_{m}, y\right)<\varepsilon .
$$


In other words, $\left\{\alpha x_{n}\right\}$ is a $\rho$-Cauchy sequence. Moreover, following the condition (iii) in Definition 2.1, we obtain $\left\{\alpha x_{n}\right\}$ is a $\rho$-Cauchy sequence for every $\alpha \in \mathbb{R}$.

\section{2-linear Operators}

Let $X$ be a real linear space. A notation $X^{2}$ is meant $X \times X$. The following definition refers to $[1,11]$.

Definition 4.1. Let $X$ and $Y$ be real linear spaces. An operator $T: X^{2}$ $\rightarrow Y$ is said to be 2-linear if for every $x, y, u, v \in X$ and $\alpha, \beta \in \mathbb{R}$, the following conditions hold:

(i) $T(x+y, u+v)=T(x, u)+T(x, v)+T(y, u)+T(y, v)$.

(ii) $T(\alpha x, \beta y)=\alpha \beta T(x, y)$.

Analogous to the definition of a 2-bounded 2-linear operator on 2-norm spaces, we define a 2- $\rho$-bounded 2-linear operator on 2-modular spaces. Let $X_{\rho}$ be a 2-modular space and $Y$ be a normed space. A 2-linear operator $T: X_{\rho}^{2} \rightarrow Y$ is said to be $2-\rho-b o u n d e d$ if there exists a real constant $M>0$ such that

$$
\|T(x, y)\| \leq M \rho(x, y)
$$

for every $x, y \in X_{\rho}$. Let us consider the following example.

Example 4.2. Let $X$ and $\rho$ be as given in Example 2.2. Note that $X_{\rho}=X$. If an operator $T: X_{\rho}^{2} \rightarrow \mathbb{R}$ is defined by

$$
T(x, y)=\left|\begin{array}{ll}
x_{1} & x_{2} \\
y_{1} & y_{2}
\end{array}\right|, \quad x, y \in X_{\rho},
$$


then we can show that $T$ is a 2-linear operator. Moreover, since

$$
|T(x, y)|=\operatorname{abs}\left(\left|\begin{array}{ll}
x_{1} & x_{2} \\
y_{1} & y_{2}
\end{array}\right|\right)=\rho(x, y)
$$

for every $x, y \in X_{\rho}, T$ is 2- $\rho$-bounded.

Let $X_{\rho}$ be a 2-modular space and $Y$ be a normed space. If $T: X_{\rho}^{2} \rightarrow Y$ is a 2- $\rho$-bounded linear operator, then it is easy to prove that $T(x, y)=0$ for every $x, y \in X_{\rho}$ which are linearly dependent. The collection of all 2- $\rho$ bounded linear operators $T: X_{\rho}^{2} \rightarrow Y$ will be denoted by $B\left(X_{\rho}^{2}, Y\right)$. It is easy to check that $B\left(X_{\rho}^{2}, Y\right)$ is a real linear space. Moreover, one can define a function $\sigma: B\left(X_{\rho}^{2}, Y\right) \rightarrow \mathbb{R}^{*}$ by

$$
\sigma(T)=\sup \left\{\frac{\|T(x, y)\|}{\rho(x, y)}: x, y \in X_{\rho}, \rho(x, y) \neq 0\right\} \text {. }
$$

The theorem below shows that the function $\sigma$ as given in (4.1) is a modular.

Theorem 4.3. The function $\sigma: B\left(X_{\rho}^{2}, Y\right) \rightarrow \mathbb{R}^{*}$ as given in (4.1) is a modular on $B\left(X_{\rho}^{2}, Y\right)$.

Proof. (i) If $T=0$, then the definition of $\sigma$ is obviously followed by $\sigma(T)=0$. Conversely, if $\sigma(T)=0$, then $T(x, y)=0$ for all $x, y \in X_{\rho}$ which are not linearly dependent. Since $T(x, y)=0$ for every $x, y \in X_{\rho}$ which are linearly dependent, we get $T(x, y)=0$ for every $x, y \in X_{\rho}$. Hence, $T=0$.

(ii) It is clear that $\sigma(-T)=\sigma(T)$ for every $T \in B\left(X_{\rho}^{2}, Y\right)$.

(iii) Take any $S, T \in B\left(X_{\rho}^{2}, Y\right)$ and $\alpha, \beta \geq 0$ such that $\alpha+\beta=1$. Then 


$$
\begin{aligned}
\sigma(\alpha S+\beta T)= & \sup \left\{\frac{\|\alpha S(x, y)+\beta T(x, y)\|}{\rho(x, y)}: \rho(x, y) \neq 0, x, y \in X_{\rho}\right\} \\
\leq & |\alpha| \sup \left\{\frac{\|S(x, y)\|}{\rho(x, y)}: \rho(x, y) \neq 0, x, y \in X_{\rho}\right\} \\
& +|\beta| \sup \left\{\frac{\|T(x, y)\|}{\rho(x, y)}: \rho(x, y) \neq 0, x, y \in X_{\rho}\right\} \\
\leq & \sigma(S)+\sigma(T) .
\end{aligned}
$$

From (i), (ii) and (iii), the assertion follows.

The following theorem states necessary and sufficient conditions so that a 2-linear operator from a 2-modular space into a normed space is 2- - -bounded.

Theorem 4.4. Let $X_{\rho}$ be a 2-modular space and $Y$ be a normed space. A 2-linear operator $T: X_{\rho}^{2} \rightarrow Y$ is 2- $\rho$-bounded if and only if there is a constant $M>0$ such that

$$
\|T(x, y)-T(u, v)\| \leq M\{\rho(x-u, y)+\rho(u, y-v)\}
$$

and

$$
\|T(x, y)-T(u, v)\| \leq M\{\rho(x-u, v)+\rho(x, y-v)\}
$$

for all $x, y, u, v \in X_{\rho}$.

Proof. ( $\Rightarrow$ :) Since $T$ is 2 - $\rho$-bounded, there exists a real constant $M>0$ such that

$$
\|T(x, y)\| \leq M \rho(x, y),
$$

for every $x, y \in X_{\rho}$. Take any $x, y, u, v \in X_{\rho}$, we have

$$
\begin{aligned}
\|T(x, y)-T(u, v)\| & =\|T(x-u, y)-T(u, y-v)\| \\
& \leq M\{\rho(x-u, y)+\rho(u, y-v)\}
\end{aligned}
$$


and

$$
\begin{aligned}
\|T(x, y)-T(u, v)\| & =\|T(x-u, v)-T(x, y-v)\| \\
& \leq M\{\rho(x-u, v)+\rho(x, y-v)\} .
\end{aligned}
$$

$(\Leftarrow:)$ It is obvious.

Theorem 4.5. Let $X_{\rho}$ be a 2-modular space and $Y$ be a normed space. If for any 2-linear operator $T: X_{\rho}^{2} \rightarrow Y, \sigma(T)$ is as defined in (4.1), then

$$
\sigma(T)=\inf \left\{M>0:\|T(x, y)\| \leq M \rho(x, y), x, y \in X_{\rho}\right\} .
$$

Proof. Since $\|T(x, y)\| \leq \sigma(T) \rho(x, y)$ for every $x, y \in X_{\rho}$,

$$
\inf \left\{M>0:\|T(x, y)\| \leq M \rho(x, y), x, y \in X_{\rho}\right\} \leq \sigma(T) \text {. }
$$

Conversely, if $K=\inf \left\{M>0:\|T(x, y)\| \leq M \rho(x, y), x, y \in X_{\rho}\right\}$, then

$$
\frac{\|T(x, y)\|}{\rho(x, y)} \leq K
$$

for every $x, y \in X_{\rho}$ with $\rho(x, y) \neq 0$. Hence, $\sigma(T) \leq K$.

Let $X_{\rho}$ be a 2-modular space and $Y$ be a normed space. An operator $T: X_{\rho}^{2} \rightarrow Y$ is said to be $(n, \rho)$-continuous at $\left(x_{0}, y_{0}\right) \in X_{\rho}^{2}$ if for every real number $\varepsilon>0$, there exists a $\delta>0$ such that for every $x, y \in X_{\rho}^{2}$ with

(i) $\rho\left(x_{0}-x, y_{0}\right)<\delta$ and $\rho\left(x, y-y_{0}\right)<\delta$, or

(ii) $\rho\left(x_{0}-x, y\right)<\delta$ and $\rho\left(x_{0}, y-y_{0}\right)<\delta$, we have $\left\|T(x, y)-T\left(x_{0}, y_{0}\right)\right\|<\varepsilon$. The operator $T$ is said to be $(n, \rho)$ continuous on $E \subset X_{\rho}^{2}$ if it is $(n, \rho)$-continuous at every $(x, y) \in E$. And $T$ is said to be $(n, \rho)$-continuous if it is $(n, \rho)$-continuous on $X_{\rho}^{2}$. 
Example 4.6. Let $X, \rho, X_{\rho}$, and $T: X_{\rho}^{2} \rightarrow \mathbb{R}$ be as given in Example 4.2. Take any $\left(x_{0}, y_{0}\right) \in X_{\rho}^{2}$. For any $(x, y) \in X_{\rho}^{2}$, we have

$$
\left|T(x, y)-T\left(x_{0}, y_{0}\right)\right| \leq \rho\left(x-x_{0}, y_{0}\right)+\rho\left(x, y-y_{0}\right)
$$

and

$$
\left|T(x, y)-T\left(x_{0}, y_{0}\right)\right| \leq \rho\left(x-x_{0}, y\right)+\rho\left(x_{0}, y-y_{0}\right) .
$$

Thus, $T$ is $(n, \rho)$-continuous at $\left(x_{0}, y_{0}\right)$.

Theorem 4.7. Let $X_{\rho}$ be a 2-modular space and $Y$ be a normed space. If a 2-linear operator $T: X_{\rho}^{2} \rightarrow Y$ is 2- $\rho$-bounded, then it is $(n, \rho)$ continuous.

Proof. By Theorem 4.4, the assertion follows.

By adding the convex property to the 2-modular $\rho$, we can prove the equivalence between 2 - $\rho$-boundedness and $(n, \rho)$-continuity of a 2-linear operator $T: X_{\rho}^{2} \rightarrow Y$. For proving this, we need the following lemma:

Lemma 4.8. Let $X_{\rho}$ be a 2-modular space and $Y$ be a normed space. A 2-linear operator $T: X_{\rho}^{2} \rightarrow Y$ is $(n, \rho)$-continuous at $(0,0) \in X_{\rho}^{2}$ if and only if for any sequence $\left\{\left(x_{n}, y_{n}\right)\right\}$ that satisfies $\lim \rho\left(x_{n}, y_{n}\right)=0$, we have $\lim \left\|T\left(x_{n}, y_{n}\right)\right\|=0$.

Proof. The proof is standard, so it is omitted.

Theorem 4.9. Let $X_{\rho}$ be a 2-modular space with $\rho$ be convex, $Y$ be a normed space, and $T: X_{\rho}^{2} \rightarrow Y$ be a 2-linear operator. The following statements are equivalent: 
3208 Burhanudin Arif Nurnugroho, Supama and Atok Zulijanto

(i) The operator $T$ is $(n, \rho)$-continuous.

(ii) The operator $T$ is $(n, \rho)$-continuous at $(0,0)$.

(iii) The set $\{\|T(x, y)\|: \rho(x, y) \leq 1\}$ is bounded.

(iv) The operator $T$ is 2- $\rho-b o u n d e d$.

Proof. (i) $\Rightarrow$ (ii) is obvious. (iv) $\Rightarrow$ (i) follows from Theorem 4.7. What remains to show are (ii) $\Rightarrow$ (iii) and (iii) $\Rightarrow$ (iv).

(ii) $\Rightarrow$ (iii) Suppose the set $\{\|T(x, y)\|: \rho(x, y) \leq 1\}$ is unbounded. Then for every $n \in \mathbb{N}$, there exists $\left(x_{n}, y_{n}\right) \in X_{\rho}^{2}$ such that $\rho\left(x_{n}, y_{n}\right) \leq 1$, but

$$
\left\|T\left(x_{n}, y_{n}\right)\right\| \geq n^{2}
$$

Set $u_{n}=\frac{x_{n}}{n}$ and $v_{n}=\frac{y_{n}}{n}$, then

$$
\rho\left(u_{n}, v_{n}\right) \leq \frac{1}{n^{2}} \rho\left(x_{n}, y_{n}\right) \leq \frac{1}{n^{2}} .
$$

This follows from the convexity of $\rho$. So, $\lim \rho\left(u_{n}, v_{n}\right)=0$. By Lemma 4.8, it must be $\lim \left\|T\left(x_{n}, y_{n}\right)\right\|=0$. However, it is impossible because

$$
\left\|T\left(u_{n}, v_{n}\right)\right\|=\frac{1}{n^{2}}\left\|T\left(x_{n}, y_{n}\right)\right\| \geq 1
$$

So, $\{\|T(x, y)\|: \rho(x, y) \leq 1\}$ is bounded.

(iii) $\Rightarrow$ (iv) By the hypothesis, there exists $M>0$ such that $\|T(x, y)\|$ $\leq M$, whenever $\rho(x, y) \leq 1$. 
Take any $(x, y) \in X_{\rho}^{2}$. It is trivial if $\rho(x, y) \leq 1$. If $\rho(x, y)>1$, then by the convexity of $\rho$,

$$
\rho\left(\frac{(x, y)}{\rho(x, y)}\right) \leq 1
$$

Hence,

$$
\|T(x, y)\| \leq M \rho(x, y)
$$

and the proof is finished.

\section{Acknowledgement}

The authors would like to thank the referees for their comments and suggestions on the manuscript.

\section{References}

[1] H. Y. Chu, S. H. Ku and D. S. Kang, Characterizations on 2-isometries, J. Math. Anal. Appl. 340 (2008), 641-628.

[2] S. Gähler, Lineare 2-normierte Räume, Math. Nachr. 28 (1964), 1-43.

[3] S. Gähler, Untersuchungen über verallgemenerte $m$-metrische Räume, I, II, III, Math. Nachr. 40 (1969), 165-189.

[4] H. Gunawan and M. Mashadi, On n-normed spaces, Int. J. Math. Math. Sci. 27 (2001), 631-639.

[5] H. Gunawan and Mashadi, On finite-dimensional 2-normed spaces, Soochow J. Math. 27(3) (2011), 321-329.

[6] M. A. Khamsi, A convexity property in modular function spaces, Department of Mathematical Sciences, The University of Texas at El Paso, 1980.

[7] S. Mazur and W. Orlicz, On some classes of linear spaces, Studia Math. 17 (1958), 97-119.

[8] J. Musielak, Orlicz spaces and modular spaces, Lecture Notes in Math., Vol. 1034, Springer-Verlag, 1983. 
3210 Burhanudin Arif Nurnugroho, Supama and Atok Zulijanto

[9] J. Musielak and W. Orlicz, On modular spaces, Studia Math. 18 (1959), 49-65.

[10] K. Nourouzi and S. Shabanian, Operator defined on $n$-modular spaces, Mediterr. J. Math. 6 (2009), 431-446.

[11] N. Srivastava, S. Bhattacharya and S. N. Lal, On Hahn-Banach extension of linear $n$-functionals in $n$-normed spaces, Math. Maced. 4 (2006), 25-32.

[12] Supama, On some common fixed point theorems in modulared spaces, Int. Math. Forum 7(52) (2012), 2571-2579. 